\title{
Expansion Formulae for Generalized Kampé De Fériet Function, Radial Wave Functios And Heat Conduction
}

\author{
Sunil Pandey ${ }^{1}$, M.K. Shukla ${ }^{2}$, P.P. Mishra ${ }^{3}$ and Rajeev Shrivastava ${ }^{4}$ \\ 1, 2 Department of Mathematics, Govt. Model Science College, Jabalpur, (M.P.), India \\ 3 Department of Mathematics, Govt. P.G. College, Panna, (M.P.), India \\ 4 Department of Mathematics, Govt. Indira Gandhi Home Science Girls College, Shahdol, (M.P.), India
}

\begin{abstract}
The object of this paper is to evaluate an improper integral involving generalized Kampé de Fériet function and then make its application to solve a boundary value problem on heat conduction. Expansion formula for generalized Kampé de Fériet function has also been obtained. A few interesting special cases along with the application of one of them in the radial wave function for the hydrogen like atoms have also been discussed.
\end{abstract}

Key Words: Generalized Kampé de Fériet function, Multiplication formula for Gamma-function, Heat conduction, Radial wave function.

\section{Introduction}

Appell's functions and the functions related to them have many applications in mathematical physics $[1,2,3]$. Singh [4] evaluated some integrals involving Kampé de Fériet function and one of them was employed to obtain a solution of a problem in heat conduction given by Bhonsle [5]. Bajpai [6], Shrivastava \& Mishra [7] have also determined the similar type of problem on heat conduction in terms of Fox's $H$-function. In continuation of the above study, the present paper is inspired by the frequent requirement of various properties of special functions which play a vital role in the study of potential theory and other allied problems in quantum mechanics. In this paper we evaluate an integral involving generalized Kampé de Fériet function and employed this to obtain a solution of a problem in heat conduction given by Bhonsle [5].

We recall the definition of generalized Kampé de Fériet function [8]:

$$
F_{l: m ; n}^{p: q ; k}\left[\begin{array}{l}
\left(a_{p}\right):\left(b_{q}\right) ;\left(c_{k}\right) ; \\
\left(\alpha_{l}\right):\left(\beta_{m}\right) ;\left(\gamma_{n}\right) ;
\end{array} x, y\right]=\sum_{r, s=0}^{\infty} \frac{\prod_{j=1}^{p}\left(a_{j}\right)_{r+s} \prod_{j=1}^{q}\left(b_{j}\right)_{r} \prod_{j=1}^{k}\left(c_{j}\right)_{s}}{\prod_{j=1}^{l}\left(\alpha_{j}\right)_{r+s} \prod_{j=1}^{m}\left(\beta_{j}\right)_{r} \prod_{j=1}^{n}\left(\gamma_{j}\right)_{s}} \frac{x^{r} y^{s}}{(r) !(s) !},
$$

where, for convergence

(i) $\quad p+q<l+m+1, \quad p+k<l+n+1, \quad|x|<\infty, \quad|y|<\infty$, or

(ii) $\quad p+q=l+m+1, \quad p+k=l+n+1, \quad$ and

$$
\left\{\begin{array}{c}
|x|^{1 /(p-l)}+|y|^{1 /(p-l)}<1, \text { if } p>l, \\
\max \{|x|,|y|\}<1, \quad \text { if } p \leq l .
\end{array}\right.
$$

Although the double hypergeometric function defined by (1.1) reduces to the Kampé de Fériet function [9] in the special case: $q=k$ and $m=n$. The generalized Kampé de Fériet function defined in (1.1) can be specialized to be expressed in terms of generalized hypergeometric series, among other things, as following instances:

$$
\begin{gathered}
F_{q: 0 ; 0}^{p: 0 ; 0}\left[\begin{array}{l}
\alpha_{1}, \cdots, \alpha_{p} \\
\beta_{1}, \cdots, \beta_{q} ; x, y
\end{array}\right]={ }_{p} F_{q}\left[\begin{array}{l}
\alpha_{1}, \cdots, \alpha_{p} ; x+y \\
\beta_{1}, \cdots, \beta_{q}
\end{array} ; . x, \alpha_{p}\right. \\
\left.F_{0: q ; s}^{0: p ; r}\left[\begin{array}{l}
\cdots ; \alpha_{1}, \cdots, \alpha_{p} ; \gamma_{1}, \cdots, \gamma_{r} \\
\cdots ; \beta_{1}, \cdots, \beta_{q} ; \delta_{1}, \cdots, \delta_{s}
\end{array} ; x, y\right]={ }_{p} F_{q}\left[\begin{array}{l}
\alpha_{1}, \cdots, \alpha_{p} \\
\beta_{1}, \cdots, \beta_{q}
\end{array}\right] x\right]{ }_{r} F_{s}\left[\begin{array}{l}
\gamma_{1}, \cdots, \gamma_{r} \\
\delta_{1}, \cdots, \delta_{s}
\end{array} ;\right] .
\end{gathered}
$$

For more details, see Srivastava and Karlsson [10, pp. 28-32].

\section{An Infinite Integral}

Here we establish the following integral to be used later in obtaining the solution of the heat conduction problem and expansion formulae.

$$
\begin{gathered}
\int_{-\infty}^{\infty} \mathrm{z}^{2 \rho} \mathrm{e}^{-z^{2}} \mathcal{H}_{2 v}(\mathrm{z}) \mathrm{F}_{1: \mathrm{m} ; \mathrm{n}}^{\mathrm{p}: \mathrm{q}}\left[\begin{array}{l}
\left(\mathrm{a}_{\mathrm{p}}\right):\left(\mathrm{b}_{\mathrm{q}}\right) ;\left(\mathrm{c}_{\mathrm{k}}\right) ; \\
\left(\alpha_{1}\right):\left(\beta_{\mathrm{m}}\right) ;\left(\gamma_{\mathrm{n}}\right) ;
\end{array} \mathrm{xz}^{2 \mathrm{~h}}, \mathrm{yz}^{2 \mathrm{~h}}\right] \mathrm{dz} \\
=2^{2 v} \mathrm{M}_{v}^{\mathrm{h}} \mathrm{F}_{1+\mathrm{h}: \mathrm{m} ; \mathrm{n}}^{\mathrm{p}+2 \mathrm{~h}: \mathrm{k}}\left[\begin{array}{l}
\left(\mathrm{a}_{\mathrm{p}}\right), \Delta(2 \mathrm{~h}, 1+2 \rho):\left(\mathrm{b}_{\mathrm{q}}\right) ;\left(\mathrm{c}_{\mathrm{k}}\right) ; \\
\left(\alpha_{\mathrm{l}}\right), \Delta(\mathrm{h}, 1+\rho-v):\left(\beta_{\mathrm{m}}\right) ;\left(\gamma_{\mathrm{n}}\right) ;
\end{array} \mathrm{xh}^{\mathrm{h}}, \mathrm{yh}^{\mathrm{h}}\right],
\end{gathered}
$$


where

(i) $\rho=0,1,2, \cdots$ and, $h$ is a positive integer,

(ii) $\quad \Delta(\mathrm{k}, \mathrm{a})$ represents $\frac{\mathrm{a}}{\mathrm{k}}, \frac{\mathrm{a}+1}{\mathrm{k}}, \cdots, \frac{\mathrm{a}+\mathrm{k}-1}{\mathrm{k}}$

and

$$
\mathrm{M}_{v}^{\mathrm{h}}=(2 \pi)^{\frac{1}{2}(1-\mathrm{h})} \mathrm{h}^{\rho} \frac{\sum_{\mathrm{j}=0}^{2 \mathrm{~h}-1} \Gamma\left(\frac{1+2 \rho+\mathrm{j}}{2 \mathrm{~h}}\right)}{\Gamma\left(\frac{1+\rho-v+\mathrm{j}}{\mathrm{h}}\right)} .
$$

To establish the integral (2.1), we first expressing generalized Kampé de Fériet function involved in its left-hand side in the series form (1.1) and then changing the order of integration and summation which is permissible under the conditions given, evaluating the z-integral with the help of

$$
\int_{-\infty}^{\infty} \mathrm{z}^{2 \rho} \mathrm{e}^{-\mathrm{z}^{2}} \mathcal{H}_{2 v}(\mathrm{z}) \mathrm{dz}=\frac{\sqrt{\pi} \cdot 2^{2(v-\rho)} \Gamma(2 \rho+1)}{\Gamma(\rho-v+1)}, \rho=0,1,2, \cdots
$$

and multiplication formula for gamma function. The formula (2.3) follows by multiplying both sides Lebdev equation [11, p. $74(4.16 .1)$ ] by $\mathrm{e}^{-\mathrm{z}^{2}} \mathcal{H}_{2 v}(\mathrm{z})$, integrating with respect to $\mathrm{z}$ from $-\infty$ to $\infty$ and using the orthogonal property of Hermite polynomials [12, pp. 192-193].

\section{Heat conduction and Generalized Kampé de Fériet function}

Hermite polynomials have been utilized by Kampé de Fériet in solving a heat conduction equation. He has obtained four theorems which are of the nature of existence theorem. Bhonsle [5, p. 356-360] has employed Hermite polynomials in solving the partial differential equation

$$
\frac{\partial \phi}{\partial t}=K \frac{\partial^{2} \phi}{\partial z^{2}}-K \phi z^{2}
$$

where $\phi(z, t)$ tends to zero for large value of $t$ and when $|z| \rightarrow \infty$, this equation is related to the problem of heat conduction given by Churchill [13, p. 130]:

$$
\frac{\partial \phi}{\partial t}=K \frac{\partial^{2} \phi}{\partial z^{2}}-h_{1}\left(\phi-\phi_{0}\right)
$$

provided that $\phi_{0}=0$ and $h_{1}=K z^{2}$.

The solution of (3.1) to be obtained here is

$$
=\sum_{r=0}^{\infty} \frac{2^{r-\frac{1}{2}} M_{r}^{h} e^{-(1+2 r) K t-z^{2} / 2}}{\sqrt{\pi}(r) !} F_{l+h: m ; n}^{p+2 h: q ; k}\left[\begin{array}{c}
\left(a_{p}\right), \Delta(2 h, 1+2 \rho):\left(b_{q}\right) ;\left(c_{k}\right) ; \\
\left(\alpha_{l}\right), \Delta\left(h, 1+\rho-\frac{r}{2}\right):\left(\beta_{m}\right) ;\left(\gamma_{n}\right) ;
\end{array} x h^{h}, y h^{h}\right] H_{r}(z) .
$$

where the conditions of validity being the same as given in (2.1).

\section{Proof :}

If we take into account all the physical considerations as given in Churchill [13, p. 130], the complete solution of (3.1) given by Bhonsle [5, p. 356-360] is

$$
\phi(z, t)=\sum_{r=0}^{\infty} Q_{r} e^{-(1+2 r) K t-z^{2} / 2} H_{r}(z)
$$

When $t=0$, let

$$
\phi(z, 0)=f(z)=z^{2 \rho} e^{-z^{2}} F_{l: m ; n}^{p: q ; k}\left[\begin{array}{l}
\left(a_{p}\right):\left(b_{q}\right) ;\left(c_{k}\right) ; x z^{2 h}, y z^{2 h} \\
\left(\alpha_{l}\right):\left(\beta_{m}\right) ;\left(\gamma_{n}\right) ;
\end{array}\right] .
$$

Now by virtue of (3.4) and (3.5), we have 


$$
z^{2 \rho} e^{-z^{2}} F_{l: m ; n}^{p: q ; k}\left[\begin{array}{l}
\left(a_{p}\right):\left(b_{q}\right) ;\left(c_{k}\right) ; \\
\left(\alpha_{l}\right):\left(\beta_{m}\right) ;\left(\gamma_{n}\right) ;
\end{array} z^{2 h}, y z^{2 h}\right]=\sum_{r=0}^{\infty} Q_{r} e^{-z^{2} / 2} H_{r}(z)
$$

Multiplying both sides of (3.6) by $H_{\mu}(z)$, integrating with respect to $z$ from $-\infty$ to $\infty$, on the left hand side using (2.1) and on the right hand side using orthogonal property of Hermite Polynomials [12, pp. 192-193], we obtain

$$
Q_{\mu}=\frac{2^{\mu-\frac{1}{2}} M_{\mu}^{h}}{\sqrt{\pi}(\mu) !} F_{l+h: m ; n}^{p+2 h: q ; k}\left[\begin{array}{c}
\left(a_{p}\right), \Delta(2 h, 1+2 \rho):\left(b_{q}\right) ;\left(c_{k}\right) ; \\
\left(\alpha_{l}\right), \Delta\left(h, 1+\rho-\frac{\mu}{2}\right):\left(\beta_{m}\right) ;\left(\gamma_{n}\right) ;
\end{array} x h^{h}, y h^{h}\right] .
$$

Substituting $Q_{r}$ from (3.7) in (3.4), we arrive at the result (3.3).

\section{Expansion Formula}

The expansion formula to be established here is

$$
\begin{aligned}
& z^{2 \rho} e^{-\frac{z^{2}}{2}} F_{l: m ; n}^{p: q ; k}\left[\begin{array}{l}
\left(a_{p}\right):\left(b_{q}\right) ;\left(c_{k}\right) ; \\
\left(\alpha_{l}\right):\left(\beta_{m}\right) ;\left(\gamma_{n}\right) ;
\end{array} z^{2 h}, y z^{2 h}\right] \\
& =\sum_{r=0}^{\infty} \frac{2^{r-\frac{1}{2}}}{\sqrt{\pi}(r) !} M_{r}^{h} F_{l+h: m ; n ; k}^{p+2 h: k}\left[\begin{array}{c}
\left(a_{p}\right), \Delta(2 h, 1+2 \rho):\left(b_{q}\right) ;\left(c_{k}\right) ; \\
\left(\alpha_{l}\right), \Delta\left(h, 1+\rho-\frac{r}{2}\right):\left(\beta_{m}\right) ;\left(\gamma_{n}\right) ;
\end{array} x h^{h}, y h^{h}\right] \mathcal{H}_{r}(z) .
\end{aligned}
$$

The conditions of validity are the same as specified for (2.1).

\section{Proof :}

The expansion (4.1) follows immediately from (3.6) and (3.7).

\section{Deductions}

In (4.1), if we take $p=l=0$, then the double hypergeometric function on the left breaks up into the product of two generalized hypergeometric functions as (1.3), thus we have

$$
\begin{aligned}
& \mathrm{z}^{2 \rho} \mathrm{e}^{-\frac{\mathrm{z}^{2}}{2}}{ }_{\mathrm{p}} \mathrm{F}_{\mathrm{q}}\left[\begin{array}{l}
\alpha_{1}, \cdots, \alpha_{\mathrm{p}} \\
\beta_{1}, \cdots, \beta_{\mathrm{q}}
\end{array} ; \mathrm{zz}^{2 \mathrm{~h}}\right] \mathrm{rF}_{\mathrm{s}}\left[\begin{array}{l}
\gamma_{1}, \cdots, \gamma_{\mathrm{r}} \\
\delta_{1}, \cdots, \delta_{\mathrm{s}}
\end{array} \mathrm{yz}^{2 \mathrm{~h}}\right] \\
& =\sum_{\mathrm{r}=0}^{\infty} \frac{2^{\mathrm{r}-\frac{1}{2}}}{\sqrt{\pi}(\mathrm{r}) !} \mathrm{M}_{\mathrm{r}}^{\mathrm{h}} \mathrm{F}_{\mathrm{h}: \mathrm{m} ; \mathrm{n}}^{2 \mathrm{~h}: \mathrm{k}}\left[\begin{array}{l}
\cdots, \Delta(2 \mathrm{~h}, 1+2 \rho):\left(\mathrm{b}_{\mathrm{q}}\right) ;\left(\mathrm{c}_{\mathrm{k}}\right) ; \\
\cdots, \Delta\left(\mathrm{h}, 1+\rho-\frac{\mathrm{r}}{2}\right):\left(\beta_{\mathrm{m}}\right) ;\left(\gamma_{\mathrm{n}}\right) ;
\end{array} \mathrm{xh}^{\mathrm{h}}, \mathrm{yh}^{\mathrm{h}}\right] \mathcal{H}_{\mathrm{r}}(\mathrm{z}),
\end{aligned}
$$

provided that the conditions easily obtainable from (2.1) are satisfied.

If, we put $\mathrm{y}=0$, in above special case, then equation (4.2) yields

$$
\begin{gathered}
\mathrm{z}^{2 \rho} \mathrm{e}^{-\frac{\mathrm{z}^{2}}{2}}{ }_{\mathrm{p}} \mathrm{F}_{\mathrm{q}}\left[\begin{array}{l}
\alpha_{1}, \cdots, \alpha_{\mathrm{p}} \\
\beta_{1}, \cdots, \beta_{\mathrm{q}}
\end{array} \mathrm{xz}^{2 \mathrm{~h}}\right] \\
=\sum_{\mathrm{r}=0}^{\infty} \frac{2^{\mathrm{r}-\frac{1}{2}} \sqrt{\pi}(\mathrm{r}) !}{M_{\mathrm{r} p+2 h}^{\mathrm{h}}} \mathrm{F}_{\mathrm{q}+\mathrm{h}}\left[\begin{array}{c}
\Delta(2 \mathrm{~h}, 1+2 \rho), \alpha_{1}, \cdots, \alpha_{\mathrm{p}} \\
\Delta\left(\mathrm{h}, 1+\rho-\frac{\mathrm{r}}{2}\right), \beta_{1}, \cdots, \beta_{\mathrm{q}}
\end{array} ; \mathrm{xh}^{\mathrm{h}}\right],
\end{gathered}
$$

which exists under conditions given in (2.1) with $\mathrm{p}=\mathrm{l}=\mathrm{y}=0$.

\section{Expansion of radial wave function}

We know from Anantharaman [14, pp. 299-301], that the normalized radial part of the wave function for the Hydrogen atom is

$$
\mathrm{R}_{\mathrm{n}, \mathrm{l}}(\gamma)=-\left[\left(\frac{2 \mathrm{z}}{n \mathrm{a}_{0}}\right)^{3} \frac{(\mathrm{n}-\mathrm{l}-1) !}{2 \mathrm{n}\{(\mathrm{n}+1) !\}^{3}}\right]^{\frac{1}{2}} \mathrm{e}^{-\frac{z \gamma}{n \mathrm{a}_{0}}}\left(\frac{2 z \gamma}{n a_{0}}\right)^{1} \mathrm{~L}_{\mathrm{n}+1}^{2 l+1}\left(\frac{2 z \gamma}{n a_{0}}\right),
$$

where the minus sign is introduced for convenience to make the function positive for small values of $\gamma$ and $L_{n+l}^{2 l+1}(z)$ is the associated Laguerre polynomials of degree $(n-l-1)$.

Further, changing the associated Laguerre polynomials in (5.1) into the confluent hypergeometric function, we obtain 


$$
R_{n, l}(\gamma)=-\left(\sqrt{\frac{z}{n^{2} a_{0}}\left(\frac{1}{\gamma^{2}}\right) \frac{(n+l) !}{(n-l-1) !}}\right) \frac{e^{\frac{z \gamma}{n a_{0}}}}{(2 l+1) !}\left(\frac{2 z \gamma}{n a_{0}}\right)^{l+1}{ }_{1} F_{1}\left[-n+l+1 ; 2 l+2 ; \frac{2 z \gamma}{n a_{0}}\right] .
$$

Now setting $c=0, p=q=h=1, a_{1}=-n+l+1, b_{1}=2 l+2, \rho=0$; replacing $z^{2}$ by $t$ and $x$ by $\frac{2 z}{n a_{0}}$ in (4.3), we get with the help of (5.2):

$$
\begin{aligned}
& R_{n, l}(t)=-\left(\sqrt{\frac{z}{n^{2} a_{0}} \frac{(n+l) !}{(n-l-1) !}}\right) \frac{e^{t\left[\frac{1}{2}-\frac{z}{n a_{0}}\right]}}{(2 l+1) !}\left(\frac{2 z}{n a_{0}}\right)^{l+1} t^{l} \sum_{r=0}^{\infty} \frac{2^{r-\frac{1}{2}} M_{r}^{1}}{\sqrt{\pi}(r) !} \\
& \text { - }{ }_{3} F_{2}\left[\begin{array}{c}
-n+l+1, \frac{1}{2}, 1 ; \quad 2 z \\
2 l+2,1-\frac{r}{2} ;
\end{array} ; H_{0}( \pm \sqrt{t})\right.
\end{aligned}
$$

where $n \geq l+1$.

The normalized radial wave functions have been calculated in Table-1 for different values of the principal quantum numbers $n$, each of which allows the azimuthal quantum number $l$ to range from 0 to $n-1$.

\begin{tabular}{|c|c|c|}
\hline$n$ & $l$ & $R_{n, l}(t)$ \\
\hline $\begin{array}{c}1 \\
\text { (k shell) }\end{array}$ & $\begin{array}{c}0 \\
\text { (1s orbital) }\end{array}$ & $-\left(\frac{z}{a_{0}}\right)^{3 / 2} e^{t\left[\frac{1}{2}-\frac{z}{a_{0}}\right]} \sum_{r=0}^{\infty} \frac{2^{r+\frac{1}{2}} M_{r}^{1}}{\sqrt{\pi}(r) !} H_{r}( \pm \sqrt{t})$ \\
\hline $\begin{array}{c}2 \\
(\mathrm{~L} \text { shell })\end{array}$ & $\begin{array}{c}0 \\
\text { (2s orbital) } \\
\end{array}$ & $-\left(\frac{z}{a_{0}}\right)^{3 / 2} e^{t\left[\frac{1}{2}-\frac{z}{2 a_{0}}\right]} \sum_{r=0}^{\infty} \frac{2^{r-1} M_{r}^{1}}{\sqrt{\pi}(r) !}{ }_{3} F_{2}\left[-1,1 / 2,1 ; \frac{z}{2,1-r / 2}\right] H_{r}( \pm \sqrt{t})$ \\
\hline $\begin{array}{c}2 \\
\text { (L shell) }\end{array}$ & $\begin{array}{c}1 \\
\text { (1p orbital) }\end{array}$ & $-\left(\frac{z}{a_{0}}\right)^{5 / 2} \frac{t}{\sqrt{3}} e^{t\left[\frac{1}{2}-\frac{z}{2 a_{0}}\right]} \sum_{r=0}^{\infty} \frac{2^{r-2} M_{r}^{1}}{\sqrt{\pi}(r) !} H_{r}( \pm \sqrt{t})$ \\
\hline $\begin{array}{c}3 \\
\text { (M shell) }\end{array}$ & $\begin{array}{c}0 \\
\text { (3s orbital) }\end{array}$ & $-\left(\frac{2 z}{3 a_{0}}\right)^{3 / 2} e^{t\left[\frac{1}{2}-\frac{z}{3 a_{0}}\right]} \sum_{r=0}^{\infty} \frac{2^{r-1} M_{r}^{1}}{\sqrt{\pi}(r) !}{ }_{3} F_{2}\left[\begin{array}{l}-2,1 / 2,1 \\
2,1-r / 2\end{array} \frac{2 z}{3 a_{0}}\right] H_{r}( \pm \sqrt{t})$ \\
\hline $\begin{array}{c}3 \\
\text { (M shell) }\end{array}$ & $\begin{array}{c}1 \\
\text { (3p orbital) }\end{array}$ & $-\left(\frac{2 z}{3 a_{0}}\right)^{5 / 2} \frac{t}{3} e^{t\left[\frac{1}{2}-\frac{2 z}{3 a_{0}}\right]} \sum_{r=0}^{\infty} \frac{2^{r-1 / 2} M_{r}^{1}}{\sqrt{\pi}(r) !}{ }_{3} F_{2}\left[-2,1 / 2,1, \frac{2 z}{4,1-r / 2} ; \frac{3 a_{0}}{3} H_{r}( \pm \sqrt{t})\right.$ \\
\hline $\begin{array}{c}3 \\
\text { (M shell) }\end{array}$ & $\begin{array}{c}2 \\
\text { (3d orbital) }\end{array}$ & $-\left(\frac{2 z}{3 a_{0}}\right)^{7 / 2} \frac{t^{2}}{15} e^{t\left[\frac{1}{2}-\frac{2 z}{3 a_{0}}\right]} \sum_{r=0}^{\infty} \frac{2^{r-\frac{5}{2}} M_{r}^{1}}{\sqrt{\pi}(r) !} H_{r}( \pm \sqrt{t})$ \\
\hline
\end{tabular}

Table - 1

RADIAL WAVE FUNCTIONS $\boldsymbol{R}_{n, l}(t)$ FOR HYDROGEN - LIKE ATOMS

\section{Conclusions}

It may be of interest that on reducing, the generalized hypergeometric functions on the left-hand side of expansions (5.2) and (5.3), yields some very interesting results. This fact is established in the light of the results [12, p. 105-106]: 
and

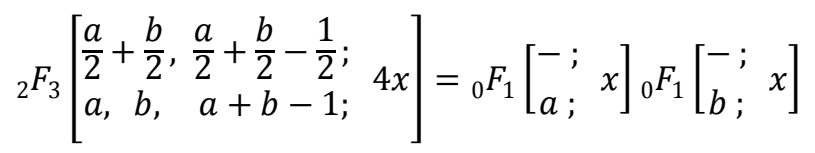

$$
{ }_{2} F_{3}\left[\begin{array}{l}
a, b-a ; \\
b, \frac{b}{2}, \frac{b}{2}+\frac{1}{2} ;
\end{array} \frac{x^{2}}{4}\right]={ }_{1} F_{1}\left[\begin{array}{l}
a ; \\
b ;
\end{array}\right]{ }_{1} F_{1}\left[\begin{array}{l}
a ;-x \\
b ;
\end{array}\right] .
$$

By proper choice of parameters, ${ }_{0} F_{1}$ can be reduce to Bessel function or transformed to ${ }_{1} F_{1}$ by Kummars second theorem [12, p. 126]. Further ${ }_{1} F_{1}$ can be reduced to Whittaker function $M_{k, h}(x)$, Hermite polynomials $H_{\mu}(x)$ and generalized Laguerre polynomials $L_{n}^{\alpha}(x)$, thereby providing us with such results as may be used in various problems encountered in Harmonic oscillator, Collision problem and Hydrogen atom [14].

It may be further remarked that generalized Kampé de Fériet function not only reduces to generalized hypergeometric function, but it also yields several other special functions [10].

\section{References}

[1] Jaeger, J.C.: A continuation formula for Appell's function $F_{1}$, J. Lond. Math. Soc., 13, (1938).

[2] Nagel, B., Olsson, P. and Weissglas, P.: Ark. Fys., 23, (1963), 137-143.

[3] Olsson, P.O.M.: A hypergeometric function of two variables of importance in perturbation theory, I,II, Ark. Fys., 30, (1965), 187191, 29, 459-465.

[4] Singh, F.: Expansion formulae for Kampé de Fériet function and radial wave function and heat conduction, Def. Sci. Jr., 21, (1970), p. 265-272.

[5] Bhonsle, B.R.: Heat conduction and Hermite Polynomials, Proc. Nat. Acad. Sci., India, 36, (1966), 356-360

[6] Bajpai, S.D.: Applications of Chebyshev-Hermite polynomials and Fox's $H$-function in heat conduction, Vijnana ParishaAnusandhan Patrika, 37, (1994), 29-36.

[7] Shrivastava, R. \& Mishra, P.P.: Expansion Formulae for Fox's $H$ - function, radial wave functions, and heat conduction (accepted for publication in the Journal Reflection des- ERA)

[8] Yang, J.: $U(n+1)$ extensions of some basic hypergeometric series identities, Adv. Stud. Contem. Math., 18, (2009), no. 2, 201-218.

[9] Kampé de Fériet, J.: Heat conduction \& Hermite Polynomials, Bull. Calcutta Math. Soc. The Golden Jubilee Commemoration Volume, (1958), 193-204.

[10] Srivastava, H.M. and Karlsson, P.W.: Multiple Gaussian hypergeometric series, Halsted Press Wiley, New York (1985).

[11] Lebdev, N.N.: Special Functions and their Applications. Prentice Hall, Inc. Engle Wood, Cliffs, N.J., (1965).

\section{Books :}

[12] Rainville, E.D.: Special functions, Chelsea Publishing Company, New York, (1960).

[13] Churchill, R.V.: Operational Mathematics. McGraw Hill, New York, (1958).

[14] Anantharaman, R.: Fundamentals of Quantum Chemistry. MacMillan India Ltd. (2001), 299-301. 